\title{
The Association Between Obstructive Sleep Apnea Defined by 3 Percent Oxygen Desaturation or Arousal Definition and Self-Reported Cardiovascular Disease in the Sleep Heart Health Study
}

\author{
Stuart F. Quan, M.D.1,2 \\ Rohit Budhiraja, M.D. ${ }^{1}$ \\ Sogol Javaheri, M.A., M.D., M.P.H. ${ }^{1}$ \\ Sairam Parthasarathy, M.D. ${ }^{2}$ \\ Richard B. Berry, M.D. ${ }^{3}$
}

'Division of Sleep and Circadian Disorders, Brigham and Women's Hospital, Harvard Medical School, Boston, MA; ${ }^{2}$ Department of Medicine, University of Arizona College of Medicine, Tucson, AZ; ${ }^{3}$ Division of Pulmonary, Critical Care, and Sleep Medicine, University of Florida, Gainesville, FL

\begin{abstract}
Background: Studies have established that OSA defined using a hypopnea definition requiring $a \geq 4 \%$ oxygen desaturation (AHI4\%) is associated with cardiovascular (CVD) or coronary heart (CHD) disease. This study determined whether OSA defined using a hypopnea definition characterized by a $\geq 3 \%$ oxygen desaturation or an arousal $(\mathrm{AHI} 3 \% \mathrm{~A})$ is associated with CVD/CHD.

Methods: Data were analyzed from 6307 Sleep Heart Health Study participants who had polysomnography. Self-reported CVD included angina, heart attack, heart failure, stroke or previous coronary bypass surgery or angioplasty. Self-reported CHD included the aforementioned conditions but not stroke or heart failure. The association between OSA and CVD/CHD was examined using logistic regression models with stepwise inclusion of demographic, anthropometric, social/behavioral and co-morbid medical conditions. A parsimonious model in which diabetes and hypertension were excluded because of their potential to be on the causal pathway between OSA and CVD/CHD also was constructed.

Results: For CVD, the odds ratios and $95 \%$ confidence intervals for $\mathrm{AHI} \% \mathrm{~A} \geq 30 /$ hour were 1.39 (1.03-1.87) and 1.45 (1.09-1.94) in the fully adjusted and parsimonious models. Results for CHD were 1.29 (0.96-1.74) and 1.36 (0.99-1.85). In participants without OSA according to more stringent $\mathrm{AHI} \%$ criteria but with OSA using the $\mathrm{AHI} \%$ A definition, similar findings were observed.

Conclusion: OSA defined using an AHI3\%A is associated with both CVD and CHD. Use of a more restrictive AHI4\% definition will misidentify a large number of individuals with OSA who have CVD or CHD. These individuals may be denied access to therapy, potentially worsening their underlying CVD or CHD.
\end{abstract}




\section{Introduction}

Obstructive sleep apnea (OSA) is a common disorder characterized by recurrent episodes of either complete upper airway collapse (apneas) or partial collapse (hypopneas) during sleep. A number of large studies have established that OSA is a risk factor for the development of hypertension and cardiovascular disease (CVD) as well as higher mortality; individuals with more severe OSA are at greater risk (1-3). The most commonly used metric of OSA severity is the apnea hypopnea index ( $\mathrm{AHI}$ ). However, there is controversy regarding the definition of the AHI. In 2012, the American Academy of Sleep Medicine (AASM) recommended that the hypopnea definition include any decrease in airflow by at least $30 \%$ from the baseline with an oxyhemoglobin desaturation of at least $3 \%$, or an arousal from sleep (4). However, several payors including the Centers for Medicare and Medicaid Services (CMS) continue to require a more stringent hypopnea definition necessitating a $4 \%$ or greater decrease in oxygen saturation (5) despite evidence documenting a relationship between the AASM recommended standard and daytime sleepiness (6). The resistance to universal acceptance of the AASM criteria is based in part on the lack of evidence that $3 \%$ desaturations or arousals have an adverse cardiovascular impact. This reluctance to adopt a more inclusive definition of sleep apnea has restricted access to OSA treatment for many patients (7). Therefore, determining if there is relationship between OSA characterized by at least $3 \%$ drop in saturation or an arousal from sleep and CVD may assist in identification of persons at risk for CVD, allow greater access to care and potentially improve other health-related outcomes.

Using the database from the Sleep Heart Health Study, a large well-characterized community based cohort that had undergone polysomnography, the current study aimed to determine the association between the AASM recommended definition of the $\mathrm{AHI}$ which incorporates hypopneas with at least a $3 \%$ desaturation or an arousal (AHI3\%A) and self-reported CVD and coronary heart disease (CHD) in middle-aged and older adults. In addition, we sought to ascertain whether there was an association between CVD or CHD and OSA severity among individuals who were not identified as having OSA using the more restrictive standard of requiring at least a $4 \%$ oxygen desaturation irrespective of an arousal (AHI4\%), but were classified as having OSA by the $\mathrm{AHI} \% \mathrm{~A}$ definition. We hypothesized that increasing OSA severity represented by the $\mathrm{AHI} \% \mathrm{~A}$ would be associated with a greater likelihood of having prevalent CVD or $\mathrm{CHD}$, and that persons who were not identified as having OSA using the AHI4\% criteria would have a higher likelihood as well.

\section{Methods}

This study analyzed data obtained from the Sleep Heart Health Study (SHHS) which was a prospective multicenter cohort study designed to investigate the relationship between OSA and cardiovascular diseases in the United States. The study's rationale and design have been published elsewhere (8). Briefly, 6,441 subjects, 40 years of age and older were recruited starting in 1995 from several ongoing "parent" cardiovascular 
and respiratory disease cohorts that were initially assembled between 1976 and 1995 (9). These cohorts included the Offspring Cohort and the Omni Cohort of the Framingham Heart Study in Massachusetts; the Hagerstown, MD, and Minneapolis, MN, sites of the Atherosclerosis Risk in Communities Study; the Hagerstown, MD, Pittsburgh, PA, and Sacramento, CA, sites of the Cardiovascular Health Study; 3 hypertension cohorts (Clinic, Worksite, and Menopause) in New York City; the Tucson Epidemiologic Study of Airways Obstructive Diseases and the Health and Environment Study; and the Strong Heart Study of American Indians in Oklahoma, Arizona, North Dakota, and South Dakota. Because of sovereignty issues, 134 participants from the Arizona cohort of the Strong Heart Study withdrew consent. Analyses were performed on the remaining 6307 participants. The SHHS was approved by each site's institutional review board for human subjects' research, and informed written consent was obtained from all subjects at the time of their enrollment.

\section{Polysomnography and Home Visit}

Participants underwent overnight in-home polysomnograms using the Compumedics Portable PS-2 System (Abbottsville, Victoria, Australia) administered by trained technicians (10). The home visits were performed by two-person, mixed-sex teams in visits that lasted 1.5 to 2 hours. Participants were asked to schedule the visit so that it would occur approximately two hours prior to their usual bedtime. At the time of the home visit, an inventory of each participant's medications was made. In addition, a health interview was completed that ascertained the presence of several health conditions. Questionnaires that were completed included the SHHS Sleep Habits Questionnaire which incorporated the Epworth Sleepiness Scale (ESS) (11) and the Medical Outcomes Study SF-36 (12). Blood pressure was measured manually in triplicate in a seated position after 5 minutes of rest (13). The average of the second and third measurements was used for this analysis. Body weight was obtained using a digital scale.

The SHHS recording montage consisted of electroencephalogram (C4/A1 and C3/A2), right and left electrooculogram, a bipolar submental electromyogram, thoracic and abdominal excursions (inductive plethysmography bands), airflow (detected by a nasaloral thermocouple (Protec, Woodinville, WA), oximetry (finger pulse oximetry [Nonin, Minneapolis, MN]), electrocardiogram and heart rate (using a bipolar electrocardiogram lead), body position (using a mercury gauge sensor), and ambient light (on/off, by a light sensor secured to the recording garment). Sensors were placed, and equipment was calibrated during an evening home visit by a certified technician. After technicians retrieved the equipment, the data, stored in real time on PCMCIA cards, were downloaded to the computers of each respective clinical site, locally reviewed, and forwarded to a central reading center (Case Western Reserve University, Cleveland, $\mathrm{OH})$. Comprehensive descriptions of polysomnography scoring and quality-assurance procedures have been previously published (14). In brief, sleep was scored according to guidelines developed by Rechtschaffen and Kales (15). Strict protocols were maintained to ensure comparability among centers and technicians. Intra-scorer and inter-scorer reliabilities were high (14). 
The apnea hypopnea index ( $\mathrm{AHI}$ ) was calculated for each participant using two definitions of hypopnea, the AASM recommended definition [AHI3\%A] and the AASM acceptable [CMS] definition [AHI4\%]. For AHI3\%A, hypopneas were identified if the amplitude of a measure of flow or volume (detected by the thermocouple or thorax or abdominal inductance band signals) was reduced discernibly (at least 30\% lower than baseline breathing) for at least 10 seconds, did not meet the criteria for apnea and the event was associated with either a 3\% oxygen desaturation from baseline or terminated with electroencephalographic evidence of an arousal. For AHI4\%, hypopneas were identified if the aforementioned reduction in flow or volume occurred and the event was associated with a $4 \%$ oxygen desaturation from baseline. In both cases, an apnea was defined as a complete or almost complete cessation of airflow, as measured by the amplitude of the thermocouple signal, lasting at least 10 seconds.

\section{Outcome Assessment}

Self-reported CVD and CHD were the outcomes of interest for this analysis and were obtained from the standardized health interview performed at the time of each participant's polysomnography home visit. Participants were asked if they had ever been told by a doctor that she or he had angina, heart attack, heart failure, or stroke and if the participant had ever undergone coronary bypass surgery or coronary angioplasty. Prevalent CVD was defined as a positive response to one or more of the aforementioned conditions or procedures. Prevalent CHD was defined as an affirmative response to the same questions with the exclusion of responses to the presence of heart failure or stroke.

\section{Covariates}

Selection of potential covariates was based on previous studies documenting an association with either CVD or CHD. These included various demographic (e.g., sex, race/ethnicity, education, marital status), anthropometric (e.g., height, weight and blood pressure [BP]), social/behavioral (e.g., smoking history, alcohol use, sleep duration, quality of life) indices as well as plasma lipids (cholesterol, high density lipoprotein [HDL], triglycerides), several diseases (depression, hypertension, diabetes) and spirometry.

The following definitions were used for those covariates that were not primarily recorded. Body mass index (BMI) was calculated as weight $(\mathrm{kg}) / \mathrm{height}\left(\mathrm{m}^{2}\right)$. The ankle arm index (AAl) was computed as the ratio of blood pressure at the ankle to that in the arm. Waist to hip ratio was the waist divided by hip circumferences. Hypertension was defined as a self-report of hypertension or the use of anti-hypertensive medications. Diabetes was considered present if it was self-reported by the participant or if there was use of oral hypoglycemic agents or insulin. Depression was defined as present if the participant indicated on the SF-36 that he/she was feeling "blue" or "down" for at least "a good bit of the time" for the previous 4 weeks, or he/she was using antidepressant medications. Insomnia was defined as often or almost always having "trouble falling asleep", "waking up during the middle of the night and having difficulty getting back to sleep" or "waking up too early in the morning and being unable to get back to sleep". 
Sleepiness was assessed by the ESS as well as by self-report of being excessively sleepy during the day most or almost all of the time.

\section{Statistical Analyses}

Mean and standard deviation, and percentages were used to provide an overall description of the data used in the analyses. Unadjusted differences were compared using Student's t test or $\chi^{2}$. For both definitions of the AHI, each participant's $\mathrm{AHI}$ was assigned to one of 4 OSA severity categories: Normal ( $\mathrm{AHI}<5$ /hour), Mild (AHI $\geq 5$ and $<15$ /hour), Moderate (AHI $\geq 15$ and $<30 /$ hour) and Severe ( $\mathrm{AHI} \geq 30 /$ hour).

Missing data was present in $4.8 \%$ of observations and were felt to be missing at random. Inasmuch as using a "complete case analysis" would result in exclusion of a significant number of participants from our analyses with a consequent reduction in statistical power, multiple imputation using the multiple imputation by chained equation (MICE) package in $\mathrm{R}$ was employed to generate replacement values. Comparison of the imputed to the original dataset did not identify any outliers in the imputed dataset and means of the same variables between datasets were comparable.

To reduce the number of relevant predictors, overfitting of models, reduce potential collinearity and minimize prediction error, a Least Absolute Shrinkage and Selection Operator (lasso) regression was performed for both outcome variables using the glmnet package in $R$. This resulted in an analytic dataset for CVD that consisted of the following: age, sex, race/ethnicity, BMI, AAI, diastolic BP, smoking, SF-36 physical component summary (PCS), SF-36 general health rating (GenHlth), SF-36 ability to perform vigorous activity (VigActiv), hypertension, diabetes, depression and HDL. For $\mathrm{CHD}$, the analytic dataset consisted of the following: age, sex, race/ethnicity, BMI, diastolic BP, smoking, PCS, GenHlth, VigActiv, hypertension, diabetes, triglycerides and HDL.

For the entire cohort, logistic regression using SPSS v27 (Armonk, NY) was used to generate increasingly complex models of the relationship between either CVD or CHD and severity of OSA adjusting for the covariates identified using the lasso regression. For CVD, after the unadjusted model, models were generated for the sequential addition of demographic factors (age, sex, race/ethnicity), anthropometric factors (BMI, AAI, diastolic BP), social/behavioral characteristics (smoking, PCS, GenHlth, VigActiv) and diseases/conditions (hypertension, diabetes, depression, HDL). For CHD after the unadjusted model, the corresponding sequential models were demographic factors (age, sex, race/ethnicity), anthropometric factors (BMI, diastolic BP), social/behavioral characteristics (smoking, PCS, GenHlth, VigActiv) and diseases/conditions (hypertension, diabetes, triglycerides, HDL). Because of the possibility that adjustment for a hypertension and a diabetes indicator would be "overadjustment" (i.e., adjustment for a variable on a causal pathway), we excluded both hypertension and diabetes from the final set of covariates in additional analyses and these are referred to as "parsimonious models." Lastly, sensitivity analyses were performed in which the natural log of AHI3\%A was used instead of categorial levels of that factor in the above models. 
Associations between both CVD and CHD, and OSA severity were further analyzed in the subgroup of participants who were not classified as having OSA based on AHI4\% criteria but were classified as OSA using AHI3\%A criteria. The moderate and severe categories were combined because of the small number of cases in the severe OSA category. Otherwise, the modelling approaches employed were identical.

In Tables 2-5, odds ratios and $95 \% \mathrm{Cl}$ are presented versus the reference level of $\mathrm{AHI}$ $<5$ /hour. $\mathrm{P}$ values refer to the overall significance of the model with respect to OSA severity. Odds ratios, $95 \% \mathrm{Cl}$ and $\mathrm{P}$ values in Table 6 refer to $\mathrm{AHI} 3 \% \mathrm{~A}$ expressed as the continuous factor $\operatorname{InAHI} \% \mathrm{~A}+0.1$ ( 0.1 added to mitigate 0 values of $\operatorname{InAHI} \% \mathrm{~A})$.

\section{Results}

Table 1 shows the univariate association of potential risk factors or characteristics with the presence of CVD or CHD.

\section{Table 1. Univariate Association of Various Characteristics to Prevalent Cardiovascular (CVD) and Coronary Heart Disease (CHD)}

\begin{tabular}{|c|c|c|c|c|}
\hline Characteristic & $\begin{array}{c}\text { CVD } \\
\text { Not Present } \\
{[N=5300)}\end{array}$ & $\begin{array}{c}\text { CVD } \\
\text { Present } \\
\text { (N=1007) }\end{array}$ & $\begin{array}{c}\text { CHD } \\
\text { Not Present } \\
\text { [N=5477] }\end{array}$ & $\begin{array}{c}\text { CHD } \\
\text { Present } \\
(\mathbb{N}=830)\end{array}$ \\
\hline & Mean (SD)/ $/ 9$ & Mean (SD)/ $/ 96$ & $\operatorname{Mean}(S D) / / 6$ & $\operatorname{Mean}(S D) / \%$ \\
\hline Age (years) & $61.8(10.9)$ & $69.2(10.0)^{\mathrm{C}}$ & $62.1(11)$ & $68.9(9.6)^{\mathrm{c}}$ \\
\hline Sex (\%male) & 44.9 & $58.9^{\mathrm{C}}$ & 45.0 & $61.6^{\mathrm{c}}$ \\
\hline Race (\% white) & 93.4 & $87.0^{6}$ & 93.3 & $86.4^{\mathrm{c}}$ \\
\hline BMI $\left(\mathrm{kg} / \mathrm{m}^{2}\right)$ & $28.0(5.2)$ & $28.4(5.0)^{\circ}$ & $28.0(5.2)$ & $28.5(5.0)$ \\
\hline Systolic BP (mm Hg) & $129(18)$ & $135(21)^{6}$ & $130(18)$ & $134(21)^{\mathrm{c}}$ \\
\hline Diastolic BP (mm Hg) & $75(11)$ & $72(11)^{6}$ & $75(11)$ & $71(11)^{\mathrm{e}}$ \\
\hline Ankle arm index & $1.12(0.12)$ & $1.10(0.16)^{c}$ & $1.12(0.12)$ & $1.11(0.15)^{c}$ \\
\hline Smoking status (\% never smokers) & 47.9 & $36.6^{\circ}$ & 47.7 & $35.3^{\mathrm{c}}$ \\
\hline SF36 Physical component score & $48(9)$ & $43(11)^{c}$ & $48(10)$ & $44(11)^{\mathrm{c}}$ \\
\hline SF36Mental component score & $53(8)$ & $53(9)^{3}$ & $53(8)$ & $53(9)^{3}$ \\
\hline SF 36.General health status ( $\% \geq$ Good) & 90.0 & $75.1^{\mathrm{c}}$ & 89.4 & $76.3^{c}$ \\
\hline SF36 Vigorous activity status (\% Not limited) & 29.7 & $15.1^{\mathrm{c}}$ & 29.2 & $15.4^{\mathrm{C}}$ \\
\hline Hypertension status (Current) & 41.8 & $80.1^{\mathrm{c}}$ & 42.2 & $81.6^{\mathrm{c}}$ \\
\hline Epworth Sleepiness Score & $7.7(4.4)$ & $8.2(4.5)^{6}$ & $7,7(4.4)$ & $8.2(4.5)^{\circ}$ \\
\hline Diabetes (Current) & 8.3 & $20.6^{c}$ & 8.6 & $21.2^{c}$ \\
\hline Depression (Current) & 10.2 & $13.4^{\circ}$ & 10.4 & $12.9^{\circ}$ \\
\hline Total cholesterol (mg/di) & $207(39)$ & $206(39)$ & $207(39)$ & $206(38)$ \\
\hline HDL cholesterol (mg/di) & $51(16)$ & $45(14)^{\mathrm{c}}$ & $51(16)$ & $46(14)^{c}$ \\
\hline Triglycerides (mg/di) & $148(99)$ & $169(111)^{c}$ & $148(99)$ & $172(1139)$ \\
\hline AHI $3 \% /$ Arousal & $16.9(15.3)$ & $21.1(16.2)^{c}$ & $17.0(15.5)$ & $21.0(15.9)^{\mathrm{c}}$ \\
\hline
\end{tabular}

$\mathrm{N}=6307$ for all characteristics except $\mathrm{AHI} 3 \% / \mathrm{A}(\mathrm{N}=6131)$

${ }^{a} p \leq 0.05 ;{ }^{b} p \leq 0.01 ;{ }^{c} p \leq 0.001$

There were 962 cases (15\%) of CVD and 797 (13\%) cases of CHD identified. Except for total cholesterol, all were either more prevalent or significantly higher or lower in participants with CVD or CHD. For both CVD and CHD, markedly higher prevalence rates were noted for sex (higher in men), hypertension, diabetes, depression, smoking 
(higher in ever smokers) and ability to engage in vigorous activity. Conversely, white race and good health status were much less common among those with CVD or CHD. Differences observed for the remaining characteristics were of lesser magnitude.

Figure 1 shows the prevalence rates of CVD or CHD as a function of OSA severity using the $\mathrm{AHI} \% \mathrm{~A}$ criteria. Both conditions were associated with increasing higher rates of disease as OSA became more severe.

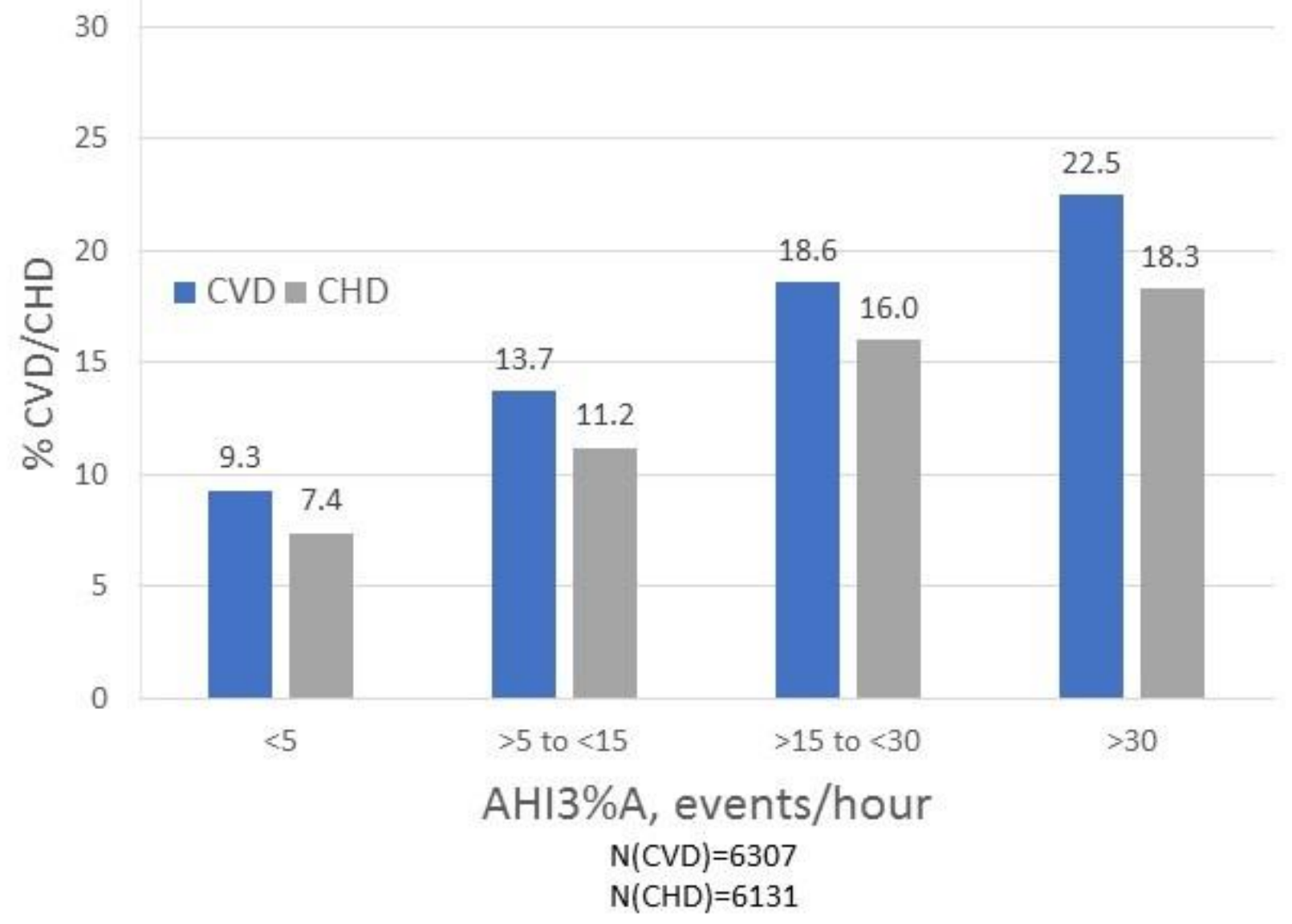

Figure 1. Percentage of participants with either cardiovascular (CVD) or coronary heart (CHD) disease according to increasing severity of obstructive sleep apnea defined using a hypopnea definition characterized by a minimum $3 \%$ oxygen desaturation or an arousal $(\mathrm{AHI} \% \mathrm{~A})$

Table 2 shows the crude and adjusted odds ratios and their $95 \%$ confidence intervals for increasing complex models of the relationship between CVD and AHI3\%A. The unadjusted model showed a strong, progressive association with increasingly severe OSA. However, as the models became increasingly complex, this relationship was attenuated and only approached statistical significance in the fully adjusted model (+Medical Conditions). Removal of hypertension and diabetes to create the 
Parsimonious model restored some of the association with a return of statistical significance.

Table 2. Adjusted Relative Odds (95\% Confidence Interval) of Self-Reported Prevalent Cardiovascular Disease According to 3\% or Arousal Apnea Hypopnea Index Severity Categories

\begin{tabular}{|c|c|c|c|c|c|}
\hline \multicolumn{6}{|c|}{ AHI $3 \%$ or Arousal Category } \\
\hline & $\leq 5$ & $\geq 5$ to $<15$ & $\geq 15$ to $<30$ & $\geq 30$ & P Value \\
\hline Unadjusted & 1.0 & $\begin{array}{c}1.55 \\
(1.23-1.97)\end{array}$ & $\begin{array}{c}2.24 \\
(1.76-2.84)\end{array}$ & $\begin{array}{c}2.85 \\
(2.21-3.67)\end{array}$ & $<0.001$ \\
\hline Demographics only" & 1.0 & $\begin{array}{c}1.13 \\
(0.88-1.44)\end{array}$ & $\begin{array}{c}1.30 \\
(1.01-1.67)\end{array}$ & $\begin{array}{c}1.54 \\
(1.17-2.02)\end{array}$ & 0.005 \\
\hline +Anthropometrics ${ }^{b}$ & 1.0 & $\begin{array}{c}1.12 \\
(0.88-1.44)\end{array}$ & $\begin{array}{c}1.28 \\
(0.98-1.65)\end{array}$ & $\begin{array}{c}1.47 \\
(1.11-1.95)\end{array}$ & 0.026 \\
\hline $\begin{array}{l}\text { +Social/Behavioral } \\
\text { Factors }\end{array}$ & 1.0 & $\begin{array}{c}1.14 \\
(0.88-1.47)\end{array}$ & $\begin{array}{c}1.31 \\
(1.00-1.70)\end{array}$ & $\begin{array}{c}1.49 \\
(1.12-1.99)\end{array}$ & 0.023 \\
\hline+ Medical Conditions $^{d}$ & 1.0 & $\begin{array}{c}1.11 \\
(0.85-1.45)\end{array}$ & $\begin{array}{c}1.25 \\
(0.95-1.65)\end{array}$ & $\begin{array}{c}1.39 \\
(1.03-1.87)\end{array}$ & 0.107 \\
\hline Parsimoniouse & 1.0 & $\begin{array}{c}1.10 \\
(0.85-1.42)\end{array}$ & $\begin{array}{c}1.29 \\
(0.98-1.68)\end{array}$ & $\begin{array}{c}1.45 \\
(1.09-1.94)\end{array}$ & 0.025 \\
\hline
\end{tabular}

aDemographics model adds age, sex, race (White vs. American Indian)

${ }^{b}$ Anthropometrics model adds BMI, Ankle Arm Index, diastolic blood pressure

'Social/Behavioral Factors model adds smoking, SF36 Physical Component Summary, SF36

General Health, SF36 Vigorous Activity

dMedical Conditions model adds hypertension, diabetes, depression and HDL

eParsimonious model includes factors in previous models, but removes hypertension and diabetes

${ }^{\mathrm{f}} \mathrm{N}=6307$

Presented in Table 3 are the models demonstrating the relationship between $\mathrm{CHD}$ and AHI3\%A.

Table 3. Adjusted Relative Odds (95\% Confidence Interval) of Self-Reported Prevalent Coronary Heart Disease According to 3\% or Arousal Apnea Hypopnea Index Severity Categories ${ }^{\dagger}$

\begin{tabular}{|c|c|c|c|c|c|}
\hline \multicolumn{6}{|c|}{ AHI $3 \%$ or Arousal Category } \\
\hline & $<5$ & $\geq 5$ to $<15$ & $\geq 15$ to $<30$ & $\geq 30$ & P Value \\
\hline Unadjusted & 1.0 & $\begin{array}{c}1.57 \\
(1.21-2.04)\end{array}$ & $\begin{array}{c}2.38 \\
(1.83-3.10)\end{array}$ & $\begin{array}{c}2.81 \\
(2.12-3.71)\end{array}$ & $<0.001$ \\
\hline Demographics only & 1.0 & $\begin{array}{c}1.14 \\
(0.87-1.49)\end{array}$ & $\begin{array}{c}1.38 \\
(1.05-1.82)\end{array}$ & $\begin{array}{c}1.48 \\
(1.10-1.99)\end{array}$ & 0.016 \\
\hline +Anthropometrics & 1.0 & $\begin{array}{c}1.13 \\
(0.86-1.49)\end{array}$ & $\begin{array}{c}1.34 \\
(1.01-1.77)\end{array}$ & $\begin{array}{c}1.40 \\
(1.03-1.90)\end{array}$ & 0.07 \\
\hline $\begin{array}{l}\text { +Social/Behavioral } \\
\text { Factors } \\
\end{array}$ & 1.0 & $\begin{array}{c}1.14 \\
(0.87-1.51)\end{array}$ & $\begin{array}{c}1.37 \\
(1.03-1.82)\end{array}$ & $\begin{array}{c}1.42 \\
(1.04-1.94)\end{array}$ & 0.053 \\
\hline +Medical Conditions & 1.0 & $\begin{array}{c}1.11 \\
(0.83-1.47)\end{array}$ & $\begin{array}{c}1.29 \\
(0.96-1.74)\end{array}$ & $\begin{array}{c}1.29 \\
(0.96-1.74)\end{array}$ & 0.222 \\
\hline Parsimonious & 1.0 & $\begin{array}{c}1.08 \\
0.82-1.43)\end{array}$ & $\begin{array}{c}1.31 \\
(0.99-1.75)\end{array}$ & $\begin{array}{c}1.36 \\
(0.99-1.85)\end{array}$ & 0.073 \\
\hline
\end{tabular}

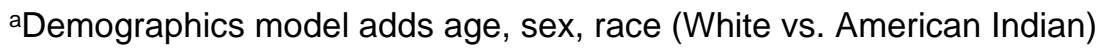

${ }^{b}$ Anthropometrics model adds BMI, diastolic blood pressure

cSocial/Behavioral Factors model adds smoking, SF36 Physical Component Summary, SF36

General Health, SF36 Vigorous Activity

dMedical Conditions model adds hypertension, diabetes, triglycerides and HDL

eParsimonious model includes factors in previous models, but removes hypertension and diabetes

${ }^{\mathrm{f}} \mathrm{N}=6307$ 
Similar to the findings for CVD, there was a progressively higher odds of having CHD as severity of OSA increased. The fully adjusted model (+Medical Conditions) was not significant, but the Parsimonious model approached statistical significance.

There were 3,326 participants who did not have OSA as defined by AHI4\% criteria. Within this cohort, 2247 were classified as OSA using the AHI3\%A criteria; 1966 $(87.4 \%)$ were mild, $271(12.0 \%)$ were moderate and $10(0.4 \%)$ were severe. For this subgroup, Table 4 presents the increasingly complex models illustrating the relationship between the presence of CVD and increasing OSA severity.

Table 4. Adjusted Relative Odds (95\% Confidence Interval) of Self-Reported Prevalent Cardiovascular Disease According to 3\% or Arousal Apnea Hypopnea Index Severity Categories in Participants Without Obstructive Sleep Apnea According to $4 \%$ Desaturation Criteria ${ }^{f}$

\begin{tabular}{|c|c|c|c|c|}
\hline \multicolumn{5}{|c|}{ AHI $3 \%$ or Arousal Category } \\
\hline & $\leq 5$ & $>5$ to $\leq 15$ & $>15$ & PValue \\
\hline Unadjusted & 1.0 & $\begin{array}{c}1.4 \overline{2} \\
(1.11-1.81)\end{array}$ & $\begin{array}{c}2.55 \\
(1.79-3.63)\end{array}$ & $<0.001$ \\
\hline Demographics only" & 1.0 & $\begin{array}{c}1.02 \\
(0.79-1.29)\end{array}$ & $\begin{array}{c}1.46 \\
(1.00-2.14)\end{array}$ & 0.091 \\
\hline +Anthropometrics & 1.0 & $\begin{array}{c}0.99 \\
(0.77-1.30)\end{array}$ & $\begin{array}{c}1.49 \\
(1.02-2.18)\end{array}$ & 0.058 \\
\hline $\begin{array}{l}\text { +Social/Behavioral } \\
\text { Factors }\end{array}$ & 1.0 & $\begin{array}{c}1.01 \\
(0.78-1.32)\end{array}$ & $\begin{array}{c}1.53 \\
(1.04-2.26)\end{array}$ & 0.053 \\
\hline +Medical Conditions & 1.0 & $\begin{array}{c}0.98 \\
(0.75-1.30)\end{array}$ & $\begin{array}{c}1.50 \\
(1.00-2.26)\end{array}$ & 0.067 \\
\hline Parsimonious ${ }^{\circ}$ & 1.0 & $\begin{array}{c}0.97 \\
(0.74-1.27)\end{array}$ & $\begin{array}{c}1.47 \\
(1.00-2.18)\end{array}$ & 0.056 \\
\hline \multicolumn{5}{|c|}{ Demographics model adds age, sex, race (White vs. American Indian) } \\
\hline \multicolumn{5}{|c|}{$\begin{array}{l}\text { 'Social/Behavioral Factors model adds smoking, SF36 Physical Component Summary, SF36 } \\
\text { General Health, SF36 Vigorous Activity }\end{array}$} \\
\hline \multicolumn{5}{|c|}{${ }^{\mathrm{d} M e d i c a l}$ Conditions model adds hypertension, diabetes, depression and HDL } \\
\hline \multicolumn{5}{|c|}{$\begin{array}{l}\text { eParsimonious model includes factors in previous models, but removes hypertension and } \\
\text { diabetes }\end{array}$} \\
\hline
\end{tabular}

Because of the relatively small number of cases with severe OSA, the moderate and severe cases were combined for these analyses. The unadjusted model showed a strong relationship with OSA severity; as model complexity increased, this finding was attenuated and only approached statistical significance in both the fully adjusted (+Medical Conditions) and Parsimonious models. As demonstrated in Table 5, similar findings were observed for CHD; the unadjusted model showed a strong association which was attenuated as the models became more complex; the fully adjusted (+medical conditions) and parsimonious models approached statistical significance. 
Table 5. Adjusted Relative Odds (95\% Confidence Interval) of Self-Reported Prevalent Coronary Heart Disease According to 3\% or Arousal Apnea Hypopnea Index Severity Categories in Participants Without Obstructive Sleep Apnea According to $4 \%$ Desaturation Criterion ${ }^{\dagger}$

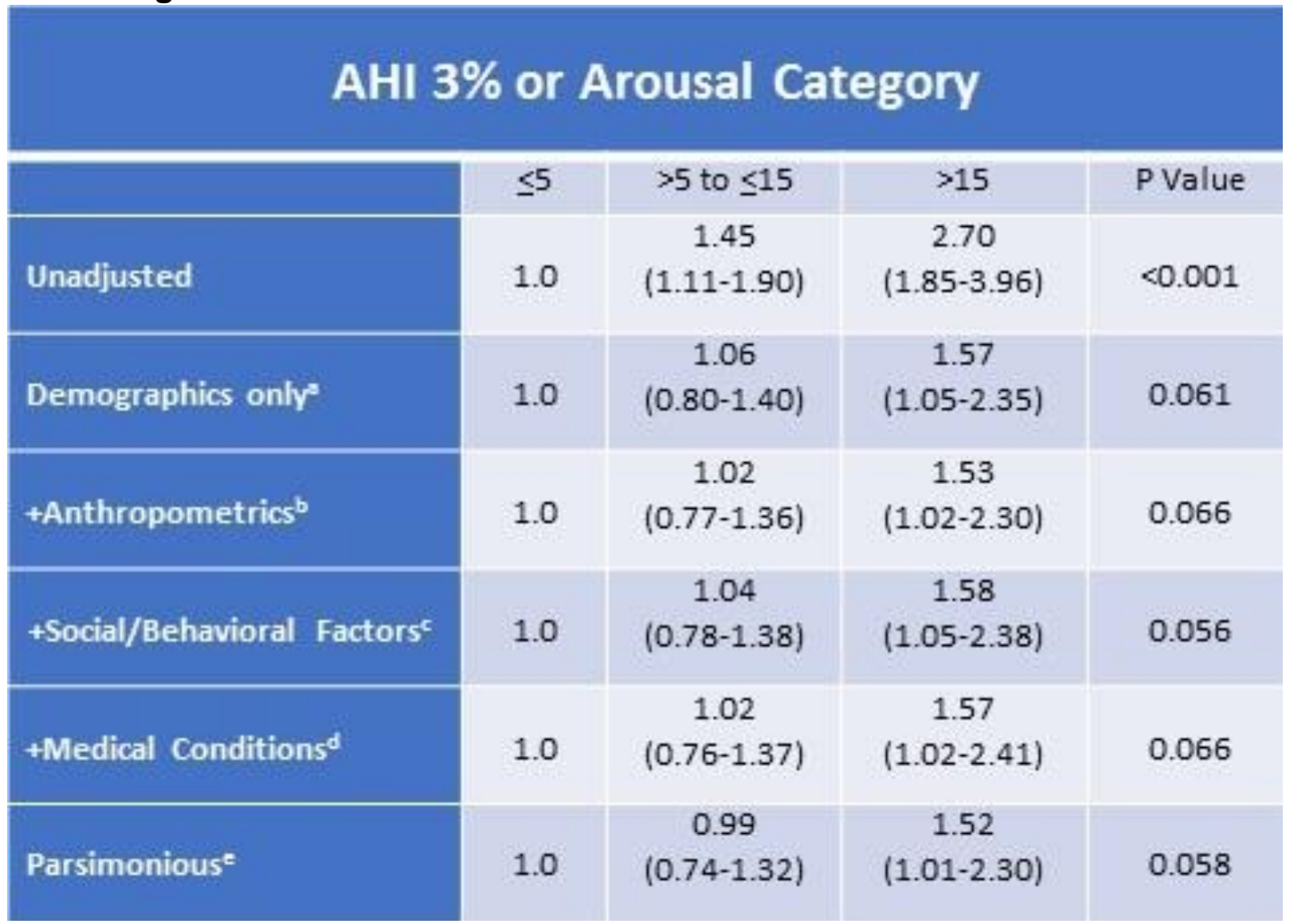

${ }^{a}$ Demographics model adds age, sex, race (White vs. American Indian)

${ }^{\mathrm{b}}$ Anthropometrics model adds BMI, diastolic blood pressure

cSocial/Behavioral Factors model adds smoking, SF36 Physical Component

Summary, SF36 General Health, SF36 Vigorous Activity

dMedical Conditions model adds hypertension, diabetes, triglycerides and HDL

eParsimonious model includes factors in previous models, but removes

hypertension and diabetes

${ }^{\dagger} \mathrm{N}=3326$

In sensitivity analyses, the natural log of $\mathrm{AHI} \% \mathrm{~A}$ was used as the index of OSA severity in lieu of a categorial representation. As shown in Table 6, in the entire cohort, for both CVD and CHD, a significant linear relationship with increasing severity of OSA was demonstrated in parsimonious models, but not the fully adjusted models. In the subgroup who did not have OSA as defined by AHI4\% criteria but did have OSA using the $A H I 3 \% A$ criteria, linear relationships noted for both CVD and CHD in the fully adjusted and parsimonious models. For CHD in the fully adjusted model, the relationship was statistically significant and approached statistical significance in the others. 
Table 6. Linear Adjusted Relative Odds (95\% Confidence Interval) of SelfReported Prevalent Cardiovascular and Coronary Heart Disease According to 3\% or Arousal Apnea Hypopnea Index Severity

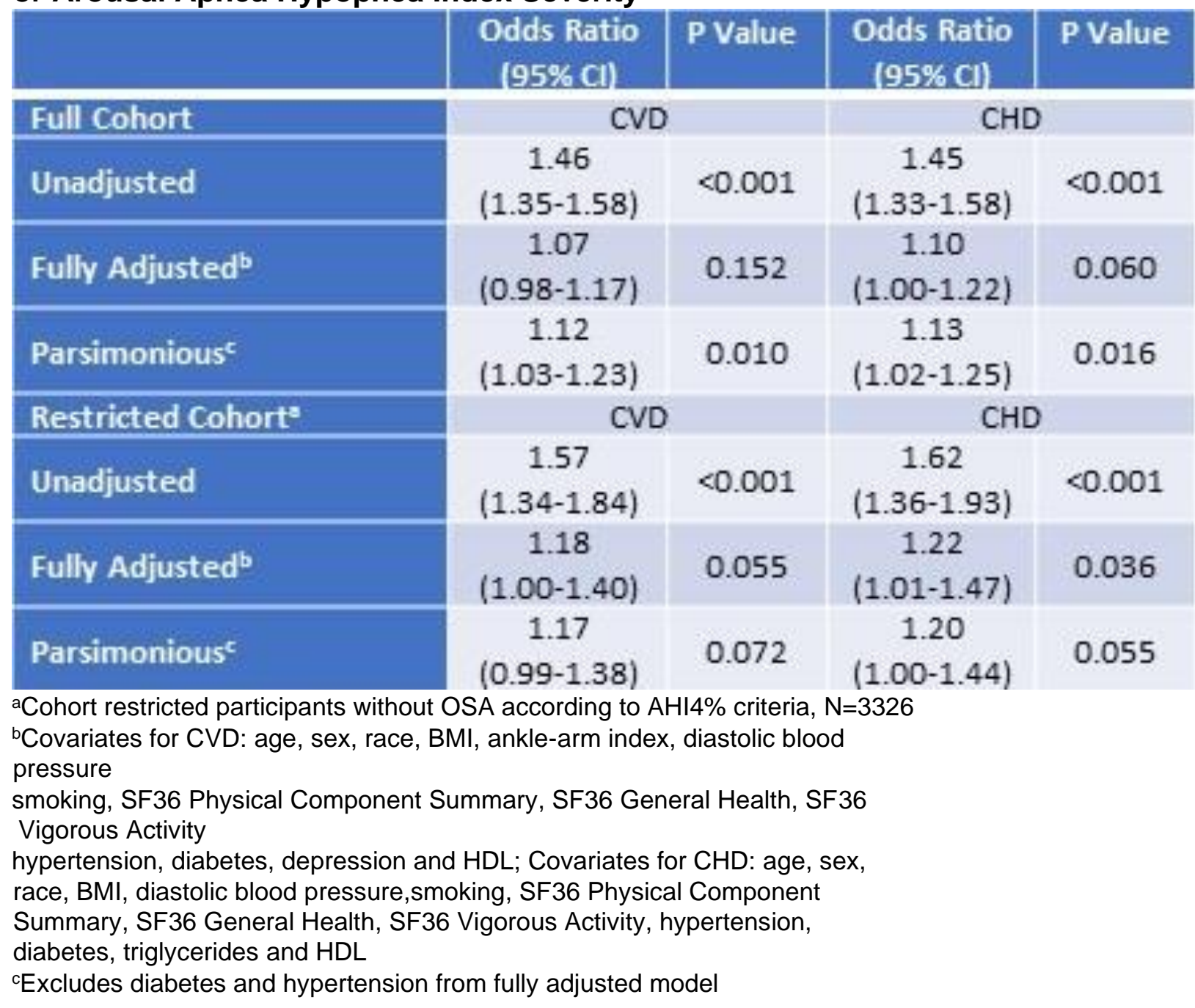

\section{Discussion}

In this large community-based study, we demonstrated that OSA defined by apneas and hypopneas characterized by $3 \%$ desaturation events or arousals is associated with an increased likelihood of self-reported CVD and CHD after controlling for a number of relevant covariates. Importantly, in a subset of this cohort who did not have OSA as defined by apneas and hypopneas requiring a minimum $4 \%$ oxygen desaturation but did have OSA using the $3 \%$ desaturation or arousal criteria, we found that the association with both CVD and CHD remained, albeit weaker. Nevertheless, our analyses overall suggest that the regulatory requirement by the Centers for Medicare and Medicaid Services (CMS) in the United States of using a 4\% desaturation definition to identify patients with OSA denies a substantial proportion of these individuals the opportunity to 
be treated for their OSA and thus reduce the risk of worsening or recurrence of their CVD or CHD.

Results from several large cohort studies including SHHS have found that OSA is associated with the presence of CVD and CHD, and that this association is stronger when the $\mathrm{AHI}$ as a metric of OSA severity increases $(1,2)$. These previous studies have used a definition of hypopnea that requires a minimum $4 \%$ oxygen desaturation (16-18). This definition has been adopted by CMS and other insurers to identify individuals as having OSA (5). However, the AASM recommends defining hypopneas with a minimum $3 \%$ desaturation or an arousal (4). This is based on evidence indicating that daytime sleepiness and other symptoms of OSA are associated with this less stringent definition of OSA (6). This distinction has important clinical implications because there are a large number of patients who do not meet the $\mathrm{AHI} \%$ criteria and but do meet the $\mathrm{AHI} 3 \% \mathrm{~A}$ criteria $(7,19)$. In the former case, they are not considered to have OSA, but do have it in the latter.

To our knowledge, our study is the first to assess the association between OSA using the $A H I 3 \% A$ criteria and CVD and CHD. We found that as OSA severity increased, there was a greater likelihood of having CVD and CHD after adjusting for a number of relevant covariates. We acknowledge that in the fully adjusted model, this association only approached statistical significance. However, in our parsimonious model which removed the presence of hypertension and diabetes, likely mediators of this relationship, the association was strengthened. Sensitivity analyses using the natural $\log$ of $\mathrm{AHI} \% \mathrm{~A}$ validated the results we observed with categories of $\mathrm{AHI}$ severity. It has been well-established that hypertension and diabetes are independent risk factors for the development of CVD and CHD. However, a number of studies have demonstrated that OSA is a risk factor for the development of both hypertension and diabetes $(1,20)$. Therefore, both of the latter conditions lie on the causal path by which OSA may increase the risk for the development of CVD and CHD. Hence, we believe that inclusion of both these conditions in our fully adjusted model may be over-adjustment and that our parsimonious model best represents the association between OSA defined by $\mathrm{AHI} \% \% \mathrm{~A}$ and CVD or CHD.

We identified there was a large subset of our cohort that had OSA using the $\mathrm{AHI} \% \mathrm{~A}$ definition, but not the AHI4\% definition of hypopnea. In this subset, we also observed an association between OSA severity and both CVD and CHD. This finding is analogous to the relationship we recently observed between OSA and the prevalence of hypertension (19). Similar to our findings with the full cohort, the fully adjusted model for both CVD and CHD was not statistically significant. However, it approached or became statistically significant in the parsimonious models. Although most of these cases were in the mild OSA category, $12.4 \%$ were moderate to severe where treatment is almost always recommended. Individuals with prevalent CVD or CHD and OSA are at risk for further complications of their CVD or CHD (21-23). However, if they do not meet the AHI4\% definition of OSA, access to OSA treatment would be denied by CMS and some insurers. 
Our findings with respect to CVD and CHD are consistent with recent analyses demonstrating that OSA defined by $\mathrm{AHI} \% \mathrm{~A}$ is associated with prevalent and incident hypertension in the SHHS cohort $(19,24)$. Similar findings also have been observed in other cohorts providing additional evidence that use of a hypopnea definition incorporating a minimum 3\% oxygen desaturation or an arousal is important in the identification of individuals with OSA (25-27).

Although there is substantial evidence emerging that intermittent hypoxemia plays an important role in the cardiovascular consequences of OSA (28), the importance of arousals remains uncertain (29). Arousals involve an increase in the sympathetic activity and a decrease in the parasympathetic activity (28) and there is some evidence linking them in the development of hypertension (30). Data from our study would suggest that they may contribute to the development of CVD or CHD as well.

Some $(31,32)$, but not all $(33)$ studies have suggested that the impact of OSA on the development of CVD or CHD is in part enhanced by the presence of sleepiness. However, in our initial assessment of potential covariates using a lasso regression, sleepiness did not emerge as a significant factor. Thus, our findings do not support the contention that sleepiness is an important factor impacting the relationship between OSA and CVD or CHD.

Our study does have a few limitations. Most important is that we identified prevalent CVD and CHD by self-report. While it is possible that some misclassification occurred, we do not think it was large. A large number of potential covariates were considered for inclusion in the models; we used a lasso regression to reduce the possibility of overadjustment and collinearity. Furthermore, the possibility of residual confounding remains. Finally, this is a cross-sectional analysis, and causality cannot be assumed.

This study has several strengths. It uses a large, well characterized cohort with the availability of data from a number of potential covariates. Additionally, the cohort had a diverse racial/ethnic, age and sex distribution. Polysomnography was used to document the presence of OSA, and not more limited sleep apnea testing.

In summary, OSA as defined by apneas and hypopneas requiring a minimum 3\% oxygen desaturation or arousal is associated with an increased likelihood of having CVD or CHD. Use of a more restrictive definition requiring a minimum $4 \%$ desaturation will misidentify a large number of individuals with OSA, and CVD or CHD. These individuals may be denied access to therapy which may prevent worsening of their underlying CVD or CHD.

\section{Acknowledgements}

SHHS acknowledges the Atherosclerosis Risk in Communities Study, the Cardiovascular Health Study, the Framingham Heart Study, the Cornell/Mt. Sinai 
Worksite and Hypertension Studies, the Strong Heart Study, the Tucson Epidemiologic Study of Airways Obstructive Diseases (TESAOD), and the Tucson Health and Environment Study for allowing their cohort members to be part of the SHHS and for sharing such data for the purposes of this study. SHHS is particularly grateful to the members of these cohorts who agreed to participate in SHHS as well. SHHS further recognizes all the investigators and staff who have contributed to its success. A list of SHHS investigators, staff, and their participating institutions is available on the SHHS website (www.jhsph.edu/shhs).

The opinions expressed in this paper are those of the authors and do not necessarily reflect the views of the Indian Health Service.

This work was supported by National Heart, Lung and Blood Institute cooperative agreements U01 HL53940 (University of Washington), U01HL53941 (Boston University), U01HL53938 (University of Arizona), U01HL53916 (University of California, Davis), U01HL53934 (University of Minnesota), U01HL53931 (New York University), U01HL53937 and U01HL64360 (Johns Hopkins University), U01HL63463 (Case Western Reserve University), U01HL63429 (Missouri Breaks Research).

SP was supported by Patient Centered Outcomes Research Institute (CER-2018C213262; PCS-1504-30430; DI-2018C2-13161; DI-2018C2-13161 COVID supplement, EADI-16493), NIH (HL126140, HL151254, Al135108, AG059202, HL158253) and American Academy of Sleep Medicine Foundation during the writing of this manuscript.

Authors' Declarations: Dr. Budhiraja reports no conflicts of interest or grant funding. Dr. Quan reports research funding from the National Institutes of Health, serves as a consultant to Jazz Pharmaceuticals, Whispersom and is a committee chair and hypopnea taskforce member for the American Academy of Sleep Medicine. Dr. Javaheri serves as a consultant for Jazz Pharmaceuticals and Harmony Biosciences. Dr. Berry reports research funding from Philips Respironics, Res Med and the University of Florida Foundation. Dr. Parthasarathy reports grants from NIH/NHLBI as PI (HL138377, HL126140; IPA-014264-00001; HL095799) or site PI (HL128954; UG3HL140144), grants from Patient Centered Outcomes Research Institute as PI (IHS-130602505; EAIN-3394-UOA) or site-investigator (PCS-1504-30430), grants from US Department of Defense as co-investigator (W81XWH-14-1-0570), grants from NIH/NCI as co-investigator (R21CA184920) and NIH/NIMHD as co-investigator (MD011600), grants from Johrei Institute, personal fees from American Academy of Sleep Medicine, non-financial support from National Center for Sleep Disorders Research of the NIH (NHLBI), personal fees from UpToDate Inc., grants from Younes Sleep Technologies, Ltd., personal fees from Vapotherm, Inc., personal fees from Merck, Inc., grants from Philips-Respironics, Inc., personal fees from Philips-Respironics, Inc., personal fees from Bayer, Inc., personal fees from Nightbalance, Inc, personal fees from Merck, Inc, grants from American Academy of Sleep Medicine Foundation (169-SR-17); In addition, Dr. Parthasarathy has a patent UA 14-018 U.S.S.N. 61/884,654; PTAS 502570970 (Home breathing device) issued. 
A preprint of this paper is available at:

medRxiv, https://doi.org/10.1101/2020.09.22.20199745

\section{Abbreviation List}

AHI

$\mathrm{AHI} \% \mathrm{~A}$

AHI4\%

AAI

BP

$\mathrm{BMI}$

$\mathrm{CHD}$

CVD

CMS

ESS

GenHlth

Glmnet

HDL

Lasso

MICE

OSA

PCS

$\mathrm{R}$

SHHS

VigActiv
Apnea Hypopnea Index

Hypopneas with at least a 3\% oxygen desaturation or an arousal

Hypopneas with at least a 4\% oxygen desaturation

Ankle arm index

Blood pressure

Body mass index

Coronary heart disease

Cardiovascular disease

Centers for Medicare and Medicaid Services

Epworth sleepiness scale

General health rating subscale of SF36

A statistical package used in $\mathrm{R}$ that fits a generalized linear model via penalized maximum likelihood

High density lipoprotein

Least Absolute Shrinkage and Selection Operator

Multiple imputation by chained equation

Obstructive sleep apnea

Physical component summary of the SF36

An open source programming language used for statistical computing and graphics

Sleep Heart Health Study

Vigorous activity rating subscale of the SF36

\section{References}

1. 1 Hou H, Zhao Y, Yu W, Dong H, Xue X, Ding J, Xing W, Wang W. Association of obstructive sleep apnea with hypertension: A systematic review and meta-analysis. $J$ Glob Health. 2018 Jun;8(1):010405. [CrossRef] [PubMed]

2. Mashaqi S, Gozal D. The impact of obstructive sleep apnea and PAP therapy on allcause and cardiovascular mortality based on age and gender - a literature review. Respir Investig. 2020 Jan;58(1):7-20. [CrossRef] [PubMed]

3. Berry RB, Budhiraja R, Gottlieb DJ, et al. Rules for scoring respiratory events in sleep: update of the 2007 AASM Manual for the Scoring of Sleep and Associated Events. Deliberations of the Sleep Apnea Definitions Task Force of the American Academy of Sleep Medicine. J Clin Sleep Med. 2012;8(5):597-619. [CrossRef] [PubMed] 
4. Anonymous. CPAP for Obstructive Sleep Apnea. Last Updated: 2020. Accessed: 09/14, 2020. https://www.cms.gov/Medicare/Coverage/Coverage-with-EvidenceDevelopment/CPAP.

5. Shamin-Uzzaman QA, Singh S, Chowdhuri S. Hypopnea definitions, determinants, and dilemmas: a focused review. Sci Sleep Pract. 2018;2(1):7. [CrossRef]

6. Raj R, Hirshkowitz M. Effect of the new Medicare guideline on patient qualification for positive airway pressure therapy. Sleep Med. 2003 Jan;4(1):29-33.

7. [CrossRef] [PubMed]

8. Quan SF, Howard BV, Iber C, Kiley JP, Nieto FJ, O'Connor GT, Rapoport DM, Redline S, Robbins J, Samet JM, Wahl PW. The Sleep Heart Health Study: design, rationale, and methods. Sleep. 1997 Dec;20(12):1077-85. [PubMed]

9. Lind BK, Goodwin JL, Hill JG, Ali T, Redline S, Quan SF. Recruitment of healthy adults into a study of overnight sleep monitoring in the home: experience of the Sleep Heart Health Study. Sleep Breath. 2003;7(1):13-24. [CrossRef] [PubMed]

10. Iber C, Redline S, Kaplan Gilpin AM, et al. Polysomnography performed in the unattended home versus the attended laboratory setting--Sleep Heart Health Study methodology. Sleep. 2004;27(3):536-540. HTTPS://PUBMED.NCBI.NLM.NIH.GOV/ 15164911 [CrossRef] [PubMed]

11. Johns MW. A new method for measuring daytime sleepiness: the Epworth sleepiness scale. Sleep. 1991;14(6):540-545. [CrossRef] [PubMed]

12. Ware JE,Jr, Sherbourne CD. The MOS 36 -item short-form health survey (SF-36). I. Conceptual framework and item selection. Med Care. 1992;30(6):473-483. [CrossRef] [PubMed]

13. O'Connor GT, Caffo B, Newman AB, et al. Prospective study of sleep-disordered breathing and hypertension: the Sleep Heart Health Study. Am J Respir Crit Care Med. 2009;179(12):1159-1164. [CrossRef] [PubMed]

14. Whitney CW, Gottlieb DJ, Redline S, et al. Reliability of scoring respiratory disturbance indices and sleep staging. Sleep. 1998;21(7):749-757. [CrossRef] [PubMed]

15. Rechtschaffen A, Kales A. A Manual of Standardized Terminology, Techniques and Scoring System for Sleep Stages of Human Subject. Washington, D.C.: US Government Printing Office, National Institute of Health Publication, 1968.

16. Marin JM, Carrizo SJ, Vicente E, Agusti AG. Long-term cardiovascular outcomes in men with obstructive sleep apnoea-hypopnoea with or without treatment with continuous positive airway pressure: an observational study. Lancet. 2005 Mar 1925;365(9464):1046-53. [CrossRef] [PubMed]

17. Shahar E, Whitney CW, Redline S, et al. Sleep-disordered breathing and cardiovascular disease: cross-sectional results of the Sleep Heart Health Study. Am J Respir Crit Care Med. 2001;163(1):19-25. [CrossRef] [PubMed]

18. Young T, Finn L, Peppard PE, Szklo-Coxe M, Austin D, Nieto FJ, Stubbs R, Hla KM. Sleep disordered breathing and mortality: eighteen-year follow-up of the Wisconsin sleep cohort. Sleep. 2008 Aug;31(8):1071-8. [PubMed]

19. Budhiraja R, Javaheri S, Parthasarathy S, Berry RB, Quan SF. The Association Between Obstructive Sleep Apnea Characterized by a Minimum 3 Percent Oxygen Desaturation or Arousal Hypopnea Definition and Hypertension. J Clin Sleep Med. 2019 Sep 15;15(9):1261-1270 [CrossRef] [PubMed] 
20. Qie R, Zhang D, Liu L, Ren Y, Zhao Y, Liu D, Liu F, Chen X, Cheng C, Guo C, Li Q, Zhou Q, Tian G, Han M, Huang S, Wu X, Qin P, Li J, Cao J, Zhang M, Huang J, Lu J, Li H, Wang J, Cheng R, Hu D. Obstructive sleep apnea and risk of type 2 diabetes mellitus: A systematic review and dose-response meta-analysis of cohort studies. J Diabetes. 2020 Jun;12(6):455-464. doi: 10.1111/1753-0407.13017. Epub 2020 Jan 22. [CrossRef] [PubMed]

21. Yudi MB, Clark DJ, Farouque O, Andrianopoulos N, Ajani AE, Brennan A, Lefkovits J, Freeman M, Hiew C, Selkrig LA, O'Brien J, Dart AM, Reid CM, Duffy SJ; Melbourne Interventional Group. Trends and predictors of recurrent acute coronary syndrome hospitalizations and unplanned revascularization after index acute myocardial infarction treated with percutaneous coronary intervention. Am Heart J. 2019 Jun;212:134-143. [CrossRef] [PubMed]

22. Lee CH, Khoo SM, Chan MY, Wong HB, Low AF, Phua QH, Richards AM, Tan HC, Yeo TC. Severe obstructive sleep apnea and outcomes following myocardial infarction. J Clin Sleep Med. 2011 Dec 15;7(6):616-21. [CrossRef] [PubMed]

23. Maia FC, Goulart AC, Drager LF, Staniak HL, Santos IS, Lotufo PA, Bensenor IM. Impact of High Risk for Obstructive Sleep Apnea on Survival after Acute Coronary Syndrome: Insights from the ERICO Registry. Arq Bras Cardiol. 2017 Jan;108(1):3137. [CrossRef] [PubMed]

24. Budhiraja R, Javaheri S, Parthasarathy S, Berry RB, Quan SF. Incidence of hypertension in obstructive sleep apnea using hypopneas defined by a 3 percent oxygen desaturation or arousal but not by only 4 percent oxygen desaturation. J Clin Sleep Med. 2020 Jul 9. Epub ahead of print. PMID: 32643602.[CrossRef] [PubMed]

25. Bouloukaki I, Grote L, McNicholas WT, Hedner J, Verbraecken J, Parati G, Lombardi C, Basoglu OK, Pataka A, Marrone O, Steiropoulos P, Bonsignore MR, Schiza SE; European Sleep Apnoea Database Network. Mild obstructive sleep apnea increases hypertension risk, challenging traditional severity classification. J Clin Sleep Med. 2020 Jun 15;16(6):889-898. [CrossRef] [PubMed]

26. Hirotsu C, Haba-Rubio J, Andries D, Tobback N, Marques-Vidal P, Vollenweider P, Waeber G, Heinzer R. Effect of Three Hypopnea Scoring Criteria on OSA Prevalence and Associated Comorbidities in the General Population. J Clin Sleep Med. 2019 Feb 15;15(2):183-194. [CrossRef] [PubMed]

27. Heinzer R, Vat S, Marques-Vidal P, Marti-Soler H, Andries D, Tobback N, Mooser V, Preisig M, Malhotra A, Waeber G, Vollenweider P, Tafti M, Haba-Rubio J.

Prevalence of sleep-disordered breathing in the general population: the HypnoLaus study. Lancet Respir Med. 2015 Apr;3(4):310-8. [CrossRef] [PubMed]

28. Sforza E, Roche F. Chronic intermittent hypoxia and obstructive sleep apnea: an experimental and clinical approach. Hypoxia (Auckl). 2016;499-108. [CrossRef] [PubMed]

29. Ryan S. Mechanisms of cardiovascular disease in obstructive sleep apnoea. J Thorac Dis. 2018;10(Suppl 34):S4201-S4211. [CrossRef] [PubMed]

30. Sulit L, Storfer-Isser A, Kirchner HL, Redline S. Differences in polysomnography predictors for hypertension and impaired glucose tolerance. Sleep. 2006;29(6):777783. [CrossRef] [PubMed] 
31. Kapur VK, Resnick HE, Gottlieb DJ, for the Sleep Heart Health Study Group. Sleep Disordered Breathing and Hypertension: Does Self-Reported Sleepiness Modify the Association? Sleep. 2008;31(8):1127-1132. [CrossRef] [PubMed]

32. Mazzotti DR, Keenan BT, Lim DC, Gottlieb DJ, Kim J, Pack Al. Symptom Subtypes of Obstructive Sleep Apnea Predict Incidence of Cardiovascular Outcomes. Am J Respir Crit Care Med. 2019;200(4):493-506. [CrossRef] [PubMed]

33. Ogilvie RP, Lakshminarayan K, Iber C, Patel SR, Lutsey PL. Joint effects of OSA and self-reported sleepiness on incident CHD and stroke. Sleep Med. 2018 Apr;44:32-37. [CrossRef] [PubMed] 